[1]

${ }^{1}$ Evidence-Based Practice Center, Robert D. and Patricia E. Kern Center for the Science of Health Care Delivery, Mayo Clinic, Rochester, MN, USA. ${ }^{2}$ Liverpool Hospital, University of New South Wales and University of Sydney, Sydney, NSW, Australia. ${ }^{3}$ Mongolian Health Initiative, Ulaanbaatar, Mongolia. ${ }^{4}$ Health Systems and Health Economics, School of Public Health, Faculty of Health Sciences, Curtin University, Perth, WA, Australia. ${ }^{5}$ School of Medicine, College of Health and Medicine, University of Tasmania, Tasmania, Australia.

\title{
Practical challenges and solutions to TB control in a lower-middle-income country: experiences from Mongolia
}

Cite as: Dobler CC, Batbayar O, Wright CM. Practical challenges and solutions to TB control in a lower-middle-income country: experiences from Mongolia. Breathe 2018; 14: 180-183.
The challenges of tuberculosis (TB) control vary significantly between countries with a low and high incidence of TB. A major focus of TB control efforts in countries with a low incidence of TB is on treatment of latent TB infection in high-risk groups [1] including contacts of patients with active TB [2], patients with medical conditions that increase the risk of TB reactivation [3-5] and migrants from settings with a high incidence of TB $[6,7]$.

By contrast, in low- and middle-income countries with a high incidence of $\mathrm{TB}$, the major focus of TB control programmes is on the detection and management of cases with active TB [1]. Frequent challenges include resource limitations, barriers to accessing TB care including distance to travel for patients to attend centralised services, indirect and direct cost associated with TB treatment for patients, limited staff capacity, and need for capacity building in health systems management and operational research. Using the example of Mongolia, we illustrate the role of operational research, community initiatives and nongovernmental organisations (NGOs) to improve TB care in low- and middle-income countries with a high incidence of TB.

\section{Operational research}

Operational research has been promoted as an important tool to improve the quality, effectiveness and reach of healthcare programmes [8]. It plays a particular role in low- and middle-income countries, where resource constraints often make it more challenging to provide effective healthcare that meets the needs of the local population. Operational research aims to detect gaps in effectiveness, efficiency and quality of local health care delivery, and to find local solutions to address these gaps [8].

The Mongolian national TB programme has recently conducted a number of operational research projects together with international collaborators [9-12]. One such project focused on the local implementation of the Xpert MTB/ RIF assay (Cepheid Inc., Sunnyvale, CA, USA), a cartridge-based nucleic acid amplification test for rapid TB diagnosis and drug susceptibility, endorsed by the World Health Organization (WHO), which was introduced in Mongolia in 2013 [9]. In this project, laboratory staff and TB physicians from Mongolia's capital Ulaanbaatar, as well as from provincial sites, 
were interviewed about their experiences with the Xpert MTB/RIF assay. The interviews were recorded, transcribed and analysed thematically. A number of barriers to Xpert MTB/RIF implementation in Mongolia were identified, highlighting areas for improvement. These included, for example, staff not being aware of the existence of Xpert MTB/RIF guidelines, lack of regular formal training on how to perform the tests, lack of local repair options for the Xpert machines and delayed treatment initiation due to poor sample quality. At the same time, enablers to Xpert MTB/RIF implementation were identified including, for example, availability of guidelines in the local language, provision of extra laboratory staff, shift working arrangements, capacity for troubleshooting internally and access to experts. Results from this study provided valuable information on how the implementation of the Xpert test can be improved in Mongolia and comparable settings.

Another operational research project was an epidemiological study of the risk of TB in pregnant women in Mongolia [10]. The project was conducted in the context of a Mongolian health policy that states that all pregnant women should be screened for active TB. Historically, chest radiography was used as the screening test but this was replaced by symptom-based screening in 2014. The study showed a 1.3-fold increased risk of TB during pregnancy compared to the general population (incidence rate ratio of $1.31,95 \% \mathrm{Cl}$ 1.08-1.59) [10], providing a rationale for the current screening strategy, given the potential for severe adverse health outcomes for the child if TB remains untreated during pregnancy. Of concern, the study revealed that $12 \%$ of pregnant women diagnosed with TB chose an abortion compared with $6 \%$ of pregnant women in the general population of Mongolia. All women with TB and an elected abortion had TB sensitive to first-line treatment. First-line drugs (isoniazid, rifampicin, ethambutol and pyrazinamide) are safe in pregnancy and are not associated with human fetal malformations. The study results indicated a need for patients and doctors to be educated about the safety of standard TB treatment in pregnancy in order to reduce abortions due to unwarranted concerns. While Mongolia has a pro-child and pro-population growth policy, the democratic government respects individuals' right to decide freely when and how many children to have. There is unrestricted access to contraceptives and first-trimester elective abortions without conditions have been legal since 1989. Abortion rates are relatively high at 16.9 per 1000 women aged $15-49$ years (2010, United Nation figures), and the government's emphasis is on preventing unintended pregnancies as well as abortions by educating its citizens about reproductive health and providing free access to family planning services.

Some organisations provide training opportunities for healthcare workers from low- and middle-income countries in order to build capacity for operational research. The Structured Operational Research and Training Initiative, a collaboration between the Special Programme for Research and Training in Tropical Diseases at WHO, the International Union Against Tuberculosis and Lung Disease, and Médecins sans Frontières, offers intensive courses in operational research that consist of three 6-day modules spaced out over 9-12 months as well as 1-day workshops and educational videos [13]. The American Thoracic Society's Methods in Epidemiologic, Clinical, and Operations Research programme offers 1-week courses in operational research at different levels of experience [14].

\section{Community initiatives}

In order to improve adherence to treatment and avoid development of drug resistance, WHO has endorsed directly observed therapy (DOT) of TB in resource-constrained settings. DOT requires health workers, community volunteers or family members to observe and record patients taking their medications. DOT is often administered in central TB clinics, which can place additional strain on these clinics [15] as well as being inconvenient for patients [16]. DOT administered in central TB clinics also results in additional direct (e.g. transport costs) and indirect costs for patients (e.g. in terms of time lost at work) [17]. Community-based models of TB care have the potential to be more patient centred and in settings where community healthcare is established for other conditions, they have the potential to facilitate integration of TB treatment with other healthcare activities [16]. A systematic review and meta-analysis of comparative effectiveness found that the odds of treatment success (a composite of treatment completion and microbiologically confirmed cure) were 1.54 times higher for community-based DOT provided by community health workers, compared with clinic-based DOT (95\% Cl 1.01-2.36) [18].

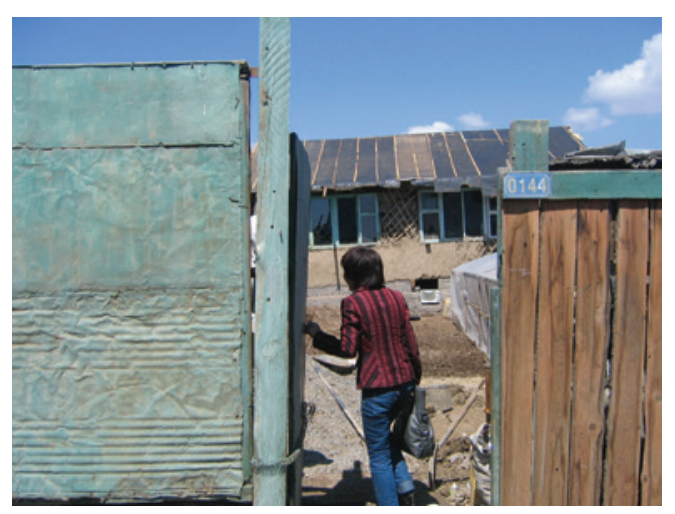

Figure 1 A MATA volunteer making a home visit. 
Limitations of the systematic review included that seven of the eight included studies were observational and that there were significant variations in study design across the six countries represented in the review.

In Mongolia, community-based DOT is provided the Mongolian Anti-Tuberculosis Association (MATA), founded in 1993 [19]. A retrospective cohort study of new sputum smear-positive patients diagnosed in 2010-2011 found a higher proportion of cured and successfully treated patients among those who received community DOT provided by MATA community volunteers compared with those who received clinic-based DOT ( $90 \%$ versus $77 \%$ for cure and $94 \%$ versus $83 \%$ for treatment success, the sum of patients with microbiologically confirmed cure and patients who completed treatment) [11].

MATA volunteers are lay health workers who collect patients' anti-TB drugs from clinics weekly and distribute these to patients at home every day Monday to Friday (with patients self-administering anti-TB drugs on Saturdays and Sundays) (figures 1 and 2). There is also a second communitybased DOT strategy, lunch DOT, where poor and malnourished TB patients receive a free meal at contracted cafeterias every weekday before taking their TB medication, providing a strong incentive for treatment adherence [11]. In addition to providing treatment support, MATA's lay health workers are trained in TB basics to provide education to patients and their families, and to participate in TB programme advocacy, communication and social mobilisation activities

Based on the success of community-based models of TB care in Mongolia, communitybased interventions are now used to spread health promotion messages designed to reduce TB transmission in Mongolia and for active screening among population groups at risk of undiagnosed TB.

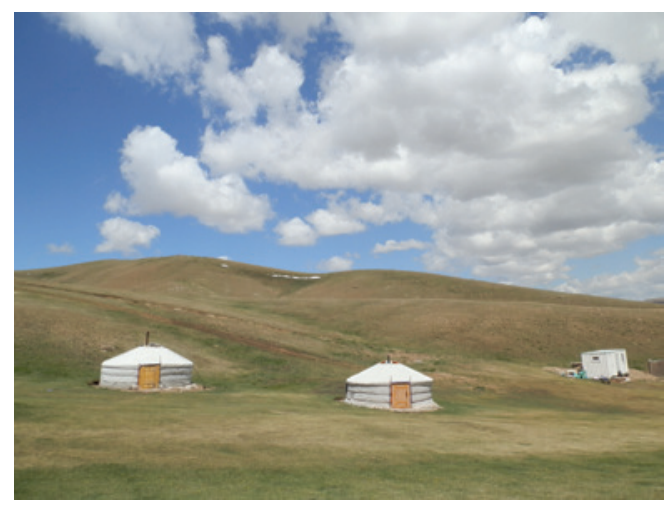

Figure 2 Mongolian gers, round tents used as dwellings.

\section{The role of NGOs}

A NGO that plays an important role in Mongolia's TB control efforts is the Mongolian Health Initiative $(\mathrm{MHI})$ and their Zero TB Mongolia team. The $\mathrm{MHI}$ is a subcontractor of the Harvard T.H. Chan School of Public Health and the Harvard School of Medicine, Boston, MA, USA. It focuses on research in the area of the association between malnourishment, in particular vitamin D deficiency, and TB [20]. The Zero TB team is an advocacy group that focuses on supporting the WHO End TB Strategy. For World TB Day on March 24, 2018, the Zero TB team launched a TB promotion campaign with the help of $>1000$ volunteers locally and abroad. MHI celebrated the World TB Day at various universities and high schools. Posters, videos and handouts promoted proper sneezing technique, and explained that TB is an infectious but curable disease. Celebrities' messages aimed at overcoming TB stigma were posted on social media and disseminated in public places. Working together with the Korean National Tuberculosis Association, 89 students received free chest radiography screening for TB. Interviews on radio and television aimed to reach people in every corner of Mongolia.

\section{Conflict of interest}

None declared.

\section{Support statement}

C.C. Dobler was supported by a fellowship from the Australian National Health and Medical Research Council (APP1123733).

\section{References}

1. World Health Organization. Guidelines on the management of latent tuberculosis infection. WHO/HTM/TB/2015.01. Geneva, WHO, 2015.

2. Dobler CC, Marks GB. Risk of tuberculosis among contacts in a low-incidence setting. Eur Respir J 2013; 41 1459-1461.

3. Dobler CC, Martin A, Marks GB. Benefit of treatment of latent tuberculosis infection in individual patients. Eur Respir J 2015 46: 1397-1406
4. Dobler CC. Biologic agents and tuberculosis. In: Schlossberg D, ed. Tuberculosis and Nontuberculous Mycobacterial Infections. 7th Edn. Washington, ASM Press, 2017; pp. 623-635.

5. Dobler CC, Cheung K, Nguyen J, et al. Risk of tuberculosis in patients with solid cancers and haematological malignancies: a systematic review and meta-analysis. Eur Respir J 2017; 50: 1700157.

6. Chan IHY, Kaushik N, Dobler CC. Post-migration follow-up of migrants identified to be at increased risk of developing 
tuberculosis at pre-migration screening: a systematic review and meta-analysis. Lancet Infect Dis 2017; 17: 770-779.

7. Dobler CC, Fox GJ, Douglas P, et al. Screening for tuberculosis in migrants and visitors from high incidence settings: present and future perspectives. Eur Respir J 2018; 52: 1800591

8. Zachariah R, Harries AD, Ishikawa N, et al. Operational research in low-income countries: what, why, and how? Lancet Infect Dis 2009; 9: 711-717.

9. Rendell NL, Bekhbat S, Ganbaatar G, et al. Implementation of the Xpert MTB/RIF assay for tuberculosis in Mongolia: a qualitative exploration of barriers and enablers. PeerJ 2017; 5: e3567.

10. Rendell NL, Batjargal N, Jadambaa N, et al. Risk of tuberculosis during pregnancy in Mongolia, a high incidence setting with low HIV prevalence. Int J Tuberc Lung Dis 2016; 20: 1615-1620.

11. Dobler CC, Korver S, Batbayar O, et al. Success of communitybased directly observed anti-tuberculosis treatment in Mongolia. Int J Tuberc Lung Dis 2015; 19: 657-662.

12. Dobler CC, Korver S, Batbayar O, et al. Multidrugresistant tuberculosis in patients for whom first-line treatment failed, Mongolia, 2010-2011. Emerg Infect Dis 2015; 21 1451-1454

13. Ramsay A, Harries AD, Zachariah R, et al. The Structured Operational Research and Training Initiative for public health programmes. Public Health Action 2014; 4 : 79-84.
14. Buist AS, Parry V. The American Thoracic Society methods in epidemiologic, clinical, and operations research program. A research capacity-building program in low- and middle-income countries. Ann Am Thorac Soc 2013; 10: 281-289.

15. Maher D, Floyd K, Sharma BV, et al. Community contribution to TB care: practice and policy review of experience of community contribution to TB care and recommendations to national TB programs. Geneva, WHO, 2003.

16. Khan MA, Walley JD, Witter SN, et al. Tuberculosis patient adherence to direct observation: results of a social study in Pakistan. Health Policy Plan 2005; 20: 354-365.

17. Khan MA, Walley JD, Witter SN, et al. Costs and costeffectiveness of different DOT strategies for the treatment of tuberculosis in Pakistan. Directly observed treatment. Health Policy Plan 2002; 17: 178-186.

18. Wright CM, Westerkamp L, Korver S, et al. Communitybased directly observed therapy (DOT) versus clinic DOT for tuberculosis: a systematic review and meta-analysis of comparative effectiveness. BMC Infect Dis 2015; 15: 210

19. Wright CM, Ng Cheong Tin P. People power: Mongolia's battle against tuberculosis. The Conversation. October 14, 2013. https://theconversation.com/ people-power-mongolias-battle-against-tuberculosis-18135

20. Ganmaa D, Munkhzul B, Fawzi W, et al. High-dose vitamin D3 during tuberculosis treatment in Mongolia. A randomized controlled trial. Am J Respir Crit Care Med 2017; 196: 628-637. 